\title{
Electrons and Phonons Cooperate in the Laser-Induced Desorption of CO from $\operatorname{Pd}(111)$
}

\author{
Maite Alducin, ${ }^{1,2, *}$ Nicholas Camillone III, ${ }^{3, \dagger}$ Sung-Young Hong, ${ }^{3, \ddagger}$ and J. Iñaki Juaristi $\circledast^{4,1,2, \S}$ \\ ${ }^{1}$ Centro de Física de Materiales CFM/MPC (CSIC-UPV/EHU), Paseo Manuel de Lardizabal 5, 20018 Donostia-San Sebastián, Spain \\ ${ }^{2}$ Donostia International Physics Center (DIPC), Paseo Manuel de Lardizabal 4, 20018 Donostia-San Sebastián, Spain \\ ${ }^{3}$ Chemistry Division, Brookhaven National Laboratory, Upton, New York 11973-5000, USA \\ ${ }^{4}$ Departamento de Física de Materiales, Facultad de Químicas (UPV/EHU), Apartado 1072, 20080 Donostia-San Sebastián, Spain
}

(Received 28 January 2019; published 10 December 2019)

\begin{abstract}
Femtosecond laser induced desorption of CO from a CO-covered Pd(111) surface is investigated with $a b$ initio molecular dynamics with electronic friction that incorporates effects due to the excited electronic and phononic systems, as well as out-of-phase coadsorbate interactions. Our simulations show evidence of an important electron-phonon synergy in promoting $\mathrm{CO}$ desorption that has largely been neglected in other similar systems. At the saturated coverage of $0.75 \mathrm{ML}$, effects due to CO-CO interadsorbate energy exchange are also important. Our dynamics simulations, in concert with site-specific desorption energy calculations, allow us to understand the large coverage dependence of the desorption yields observed in experiments.
\end{abstract}

DOI: 10.1103/PhysRevLett.123.246802

Femtosecond laser pulses in the ultraviolet, visible, and near-infrared efficiently drive surface adsorbate dynamical processes, including diffusion, desorption, and reactions [1-4]. With large fluences $\left(\sim 1 \mathrm{~mJ} / \mathrm{cm}^{2}\right)$ incident on metal surfaces - which absorb a sizable fraction of the light at these wavelengths - the excitation of adsorbates occurs indirectly. The process is initiated with the excitation of the electronic system that is driven out of equilibrium by the laser radiation. Subsequently, the photon-excited electronic system can transfer energy directly to the adsorbate, but also indirectly via the excited surface lattice that results from electron-phonon coupling. Two-pulse correlation (TPC) experiments are usually employed to establish the timescale of the adsorbate-substrate energy transfer and, accordingly, determine whether the adsorbate reaction is electron or phonon mediated [1]. Most systems investigated so far are characterized by time responses of a few picoseconds or less that are ascribed to a dominant electron-assisted process [5-9]. Only the desorption of $\mathrm{CO}$ from $\mathrm{Ru}(0001)$, with a TPC time response of $\sim 20 \mathrm{ps}$, is considered to be phonon dominated [10,11], as confirmed theoretically [12] using the 12-dimensional Langevin dynamics model proposed in Ref. [13]. Thus it is fair to conclude that the role of the electron-excited phonons has generally been underappreciated, due in part to the lack of a theoretical model advanced enough to incorporate all the ingredients in this complex scenario [2,3].

In this Letter, we incorporate time-dependent electronic and phononic temperatures within the $a b$ initio molecular dynamics with electronic friction (AIMDEF) scheme [1420] in order to include all substrate-mediated effects in the adsorbate dynamics. Also, coadsorbate interactions that are identified as crucial in other systems $[8,20,21]$ are considered. Our simulations show that both electrons and phonons are critical to understanding the ultrafast desorption dynamics of $\mathrm{CO}$ from $\mathrm{Pd}(111)$. In particular, we show that the electron-excited phonons enhance the efficiency of the electron-mediated desorption mechanism by reducing the $\mathrm{CO}$-surface bond strength. Interestingly, experiments investigating $\mathrm{CO}$ desorption at different coverages reported a $\sim 100$-fold increase of the desorption yield with the $\mathrm{CO}$ coverage varying between 0.24 and $0.75 \mathrm{ML}$ [22] for a laser fluence $F=7.66 \mathrm{~mJ} / \mathrm{cm}^{2}$. This finding constitutes a much stronger coverage dependence than previously observed for any other adlayer-metal system, for which factors $\leq 3$ were reported [11,23-25]. Our simulations, which allow us to treat the coverage dependent dynamics, are finally able to qualitatively reproduce and explain the reasons behind the strong coverage dependent desorption.

The main lines of our proposed methodology, hereafter denoted as $\left(T_{e}, T_{l}\right)$-AIMDEF, are as follows (see Supplemental Material [26] for details). Following Refs. [44,45], the adsorbate dynamics in a highly excited electronic environment is described by evaluating classical trajectories of the adsorbates by solving a Langevin equation in the ground state potential energy surface. The coupling of the adsorbate to the laser-excited electronic system is incorporated via electronic friction and stochastic forces that arise from a time-dependent electronic temperature $T_{e}(t)$. The latter is calculated within the twotemperature model (2TM) that specifically describes the response of the metal to the laser pulse in terms of coupled hot electronic and phononic excitations characterized by two distinct time-dependent temperatures $T_{e}(t)$ and $T_{l}(t)$ [46]. This model and its variants have successfully been 
applied to a variety of adsorbate-surface combinations, provided that the adsorbate-surface interaction is accurately described at the density functional theory (DFT) level [12,47-49]. The desorption of $\mathrm{H}_{2}$ [49] and $\mathrm{CO}$ [12] from $\mathrm{Ru}(0001)$ are good examples of the agreement achieved by this model with available experiments. The theoretical justification for its validity is that due to the typical short lifetime of the electronic excited states of adsorbates at metal surfaces (of the order of few fs), the trajectories propagate on the ground potential energy surface most of the time [47-49]. Inverse photoemission electron spectroscopy experiments show that the lifetime of the $2 \pi^{*}$ resonance of $\mathrm{CO}$ on $\mathrm{Pd}(111)$ is in the range 0.240.44 fs [50-53], which supports the use of this methodology in our case. In the present work, we rely on the AIMDEF method [14-20], in which the electronic friction coefficients acting on the adsorbates are obtained within the local density friction approximation (LDFA) [54,55]. More precisely, we use the recently adapted AIMDEF that includes random forces that depend on a time dependent $T_{e}(t)$ [20]. In this way, the Langevin equation is integrated calculating on the fly the adiabatic, friction, and stochastic forces. As a further improvement, we employ time-dependent thermostats in order to incorporate effects due to the excited phonons indirectly created by the laser pulse. In our simulations, the Pd atoms in the first two surface layers are coupled to a Nosé-Hoover thermostat $[56,57]$ with the time dependent $T_{l}$ obtained in the 2TM.

Simulations of the desorption dynamics are performed for 0.33 and $0.75 \mathrm{ML}$ coverages. At $0.33 \mathrm{ML}$, the CO molecules adsorb on threefold hollow fcc sites [58-64], while they are equally distributed in the top and threefold hollow fcc and hcp sites at the saturation coverage of 0.75 ML [59,62-64]. The 0.33 and $0.75 \mathrm{ML}$ adlayers are, respectively, described with a $(3 \times 3)$ cell in the surface plane that contains three $\mathrm{CO}$ molecules adsorbed at the fcchollow sites and a $(4 \times 2)$ cell with six $\mathrm{CO}$ adsorbed at two top, two hcp-hollow, and two fcc-hollow sites (see Fig. 1, top panel). These large cells allow us to account for out of phase movements of the coadsorbed $\mathrm{CO}$ molecules and to investigate the effects of the interadsorbates interactions, as done previously for $\mathrm{H} / \mathrm{Ru}(0001)$ [20]. All calculations are performed with VASP $[65,66]$ (version 5.4 and our AIMDEF module [14-20]) using DFT and the vdW-DF exchangecorrelation (xc) functional proposed by Dion et al. [67] (see Ref. [26] for all computational details).

The desorption energy is one of the important factors influencing the desorption probability. Figure 1 (top) shows the interaction energy between each individual (upright) $\mathrm{CO}$ molecule and the (remaining) CO-covered $\mathrm{Pd}(111)$ surface calculated at different heights above its corresponding adsorption site (see Supplemental Material [26]). The experimental adsorption energies estimated in Ref. [22] from TPD measurements are $E_{a}=-1.38,-0.78$, and $-0.5 \mathrm{eV}$ for $0.24,0.64$, and $0.75 \mathrm{ML}$, respectively.
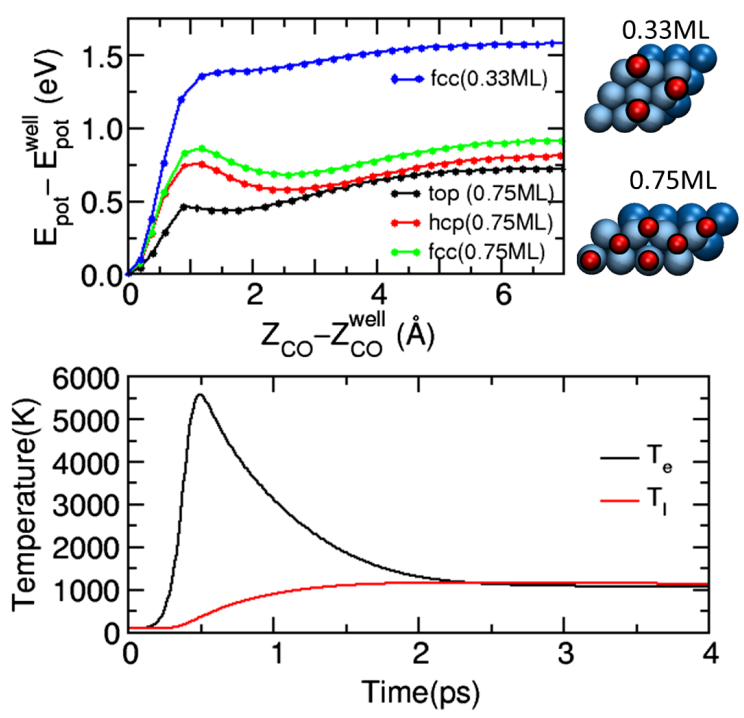

FIG. 1. Top panel: Surface unit cells used in the AIMDEF simulations for 0.33 and $0.75 \mathrm{ML}$ (right). Potential energy as a function of the $\mathrm{CO}$ geometrical-center height $Z_{\mathrm{CO}}$ to the bottom of its corresponding adsorption well $Z_{\mathrm{CO}}^{\mathrm{well}}$ for each coverage and adsorption site (left plot). Bottom panel: Electronic and phononic temperatures calculated from the $2 \mathrm{TM}$.

Assuming a linear dependence of the adsorption energy on coverage, an experimental value of around $-1.2 \mathrm{eV}$ can be estimated for desorption from the fcc hollows at $0.33 \mathrm{ML}$. The experimental value of $-0.5 \mathrm{eV}$ at 0.75 is best interpreted as representing desorption from the top site [22]. These values can be compared with those derived from our DFT calculations: $E_{a} \approx-1.58 \mathrm{eV}$ at $0.33 \mathrm{ML}$ (fcc hollow) and $E_{a} \approx-0.73 \mathrm{eV}$ for the top-site $\mathrm{CO}$ at $0.75 \mathrm{ML}$. Even if the vdW-DF functional reduces the usual COsurface overbinding obtained with xc functionals based on the generalized gradient approximation [68], our calculations still overestimate the experimental adsorption energies by $\sim 0.38 \mathrm{eV}$ for $0.33 \mathrm{ML}$ and $\sim 0.23 \mathrm{eV}$ for $0.75 \mathrm{ML}$. The consequences of this fact will be discussed below.

We run AIMDEF simulations for an absorbed fluence $F=13 \mathrm{~mJ} / \mathrm{cm}^{2}$. Figure 1 (bottom) shows the time dependence of $T_{e}$ and $T_{l}$ obtained from the 2TM for a $780 \mathrm{~nm}$ wavelength and $100 \mathrm{fs} \mathrm{sech}^{2}$ laser pulse excitation of $\mathrm{Pd}(111)$ initially at $90 \mathrm{~K}$ [69]. The surface temperature defined in terms of the kinetic energy of the Pd atoms follows closely $T_{l}$ for the two coverages (See Supplemental Material [26]). Table I shows the $\left(T_{e}, T_{l}\right)$-AIMDEF simulated desorption probabilities per $\mathrm{CO}$ molecule for the two coverages. In each case, we run 100 trajectories with a simulation time of $3.5 \mathrm{ps}$ each. The probabilities per molecule are obtained by dividing the total number of desorbing molecules by the total number of trajectories (100) and the number of adsorbed molecules in each simulation cell ( 3 for 0.33 and 6 for $0.75 \mathrm{ML}$ ).

The first important observation is the strong coverage dependence that amounts to a factor $\sim 50$, a result similar in 
TABLE I. Desorption probabilities obtained at 0.33 and 0.75 ML from different kinds of AIMDEF simulations (see text) and $F=13 \mathrm{~mJ} / \mathrm{cm}^{2}$. For $0.75 \mathrm{ML}$, the total number of desorbing molecules from each initial adsorption site is also specified (there are 200 molecules of each type with the 100 simulated trajectories).

\begin{tabular}{lccrrr}
\hline \hline Simulation & $0.33 \mathrm{ML}$ & $0.75 \mathrm{ML}$ & top & hcp & fcc \\
\hline$\left(T_{e}, T_{l}\right)$-AIMDEF & 0.003 & 0.143 & 40 & 24 & 22 \\
$T_{e}$-AIMDEF & 0 & 0.044 & 24 & 2 & 1 \\
$T_{l}$-AIMD & 0.003 & 0.055 & 16 & 8 & 8 \\
$T_{e}^{\text {FHW }}$-AIMDEF & $\cdots$ & 0.01 & 6 & $\cdots$ & $\cdots$ \\
\hline \hline
\end{tabular}

magnitude to that determined experimentally [22]. As already remarked, this dramatic increase of the desorption probability with coverage was regarded as characteristic of this system [22]. The obtained factor $\sim 50$ must be taken with care, because only one desorption event takes place for $0.33 \mathrm{ML}$, which implies that the statistical uncertainty for this coverage is large. However, note that in other systems [11,23-25] the experimental measurements showed that coverage dependencies are below a factor of 3 . In spite of the statistical uncertainty, we can exclude such low values of the coverage dependence from our simulations. For this reason, we can safely conclude that the unusual strong coverage dependence found in the $\mathrm{CO} / \mathrm{Pd}(111)$ system, though qualitatively, is well captured by our simulations. Additionally, we cannot expect quantitative agreement with the measured desorption yields due to the overestimation of the desorption energy barriers in our DFT calculations. Instead, to account for the larger barriers, we have run our simulations at a larger fluence than the representative $\sim 8 \mathrm{~mJ} / \mathrm{cm}^{2}$ value that was used to compare desorption probabilities across the full coverage range in Ref. [22]. Thus, the degree of surface excitation is greater in the simulations, but the simulated probabilities are in general agreement with the experimental results $(0.003$ and 0.3 at 0.33 and $0.75 \mathrm{ML}$, respectively [22]).

Our simulations also reveal the binding site dependent desorption that exists at $0.75 \mathrm{ML}$, information that is hidden in experiments. The results from $\left(T_{e}, T_{l}\right)$ AIMDEF in Table I show that desorption is dominated by molecules at the top site, but that desorption from the hollow sites is also important. Particularly, $46.5 \%$ of the desorbed molecules were initially at the top site, $27.9 \%$ of them in the hcp site, and $25.6 \%$ of them in the fcc site.

The time evolution of the mean kinetic energies $\left\langle E_{\mathrm{kin}}\right\rangle$ of the nondesorbing adsorbates (Fig. 2) indicates that the aforementioned coverage and site-dependent differences in desorption barriers-and not differences in the substrate electron-adsorbate coupling strengths - are primarily responsible for the coverage and adsorption site dependence of the desorption probabilities. Specifically, the $\left\langle E_{\mathrm{kin}}\right\rangle$ gains for fcc-CO at $0.33 \mathrm{ML}$ and the $\mathrm{CO}$ at all three sites at

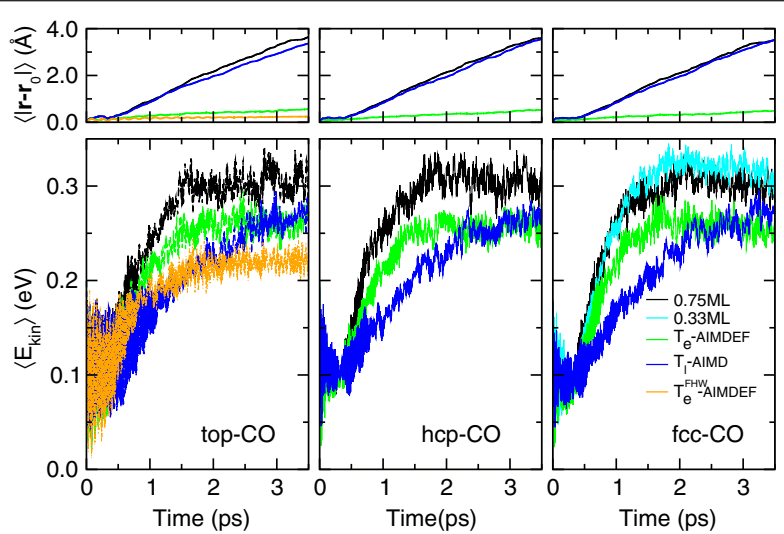

FIG. 2. Bottom: Mean kinetic energy of the nondesorbing CO against time for each coverage and adsorption site: top CO (left panel), hcp CO (middle panel), and fcc CO (right panel). Results obtained from $\left(T_{e}, T_{l}\right)$-AIMDEF for 0.75 (black) and $0.33 \mathrm{ML}$ (light blue); and from $T_{e}$-AIMDEF (green), $T_{l}$-AIMD (blue), and $T_{e}^{\mathrm{FHW}}$-AIMDEF (orange) for $0.75 \mathrm{ML}$. Top: Mean distance of the nondesorbing-CO geometrical center with respect to its initial position at $0.75 \mathrm{ML}$. Color criteria and ordering of panels as in bottom figures.

0.75 ML are all very similar. However, the desorption probabilities (Table I) are strongly correlated with the desorption energies (Fig. 1). We find that the 0.33 ML fcc $\mathrm{CO}$ exhibits a slightly larger $\left\langle E_{\text {kin }}\right\rangle$ gain, attributable to their being bound more strongly and closely to the surface. This attribution is borne out by results of friction coefficient calculations (see Supplemental Material [26]). Similarly, we see a relatively slow rise in $\left\langle E_{\text {kin }}\right\rangle$ for the top site $\mathrm{CO}$, attributable to their being more loosely bound and farther from the surface, where the electron density is lower. But these coverage and site dependencies in coupling strengths do not result in significant differences in $\left\langle E_{\text {kin }}\right\rangle$ over the $\sim 3$ ps timescale relevant to desorption, and therefore cannot account for the large coverage and site dependencies of the desorption probabilities (Table I). We can conclude, therefore, that it is the differences in desorption barrier height (Fig. 1) that is ultimately responsible for governing the desorption probability.

By performing two additional types of simulations we are able to disentangle the contribution of the heated electronic and phononic systems in the desorption dynamics that could not be unambiguously extracted by a phenomenological 2TM analysis of the TPC measurements [22]. The effect of the excited electrons is isolated in the $T_{e}$-AIMDEF simulations by fixing the Pd atoms to their initial positions. On the contrary, only the effect of the phononic system is included in the $T_{l}$-AIMD simulations by setting the friction coefficients equal to zero. Table I shows that the desorption probabilities obtained with $T_{e^{-}}$ AIMDEF and $T_{l}$-AIMD at $0.75 \mathrm{ML}$ are rather similar, but about a factor 3 and 2.6 smaller than in $\left(T_{e}, T_{l}\right)$-AIMDEF, respectively. These results demonstrate that both the photon-induced hot electrons and concomitant hot phonons 
contribute efficiently to $\mathrm{CO}$ desorption at saturation coverage. Furthermore, the total probability for desorption when both mechanisms are included is larger than the sum of the probabilities for each independent mechanism, indicating a synergistic effect. In this respect, it would be interesting to investigate possible synergistic effects in other similar systems that up to now were considered as electrondominated [5-9,70]. Note in passing that although the $T_{e}$-AIMDEF and $T_{l}$-AIMD desorption probabilities are very similar, Fig. 2 shows that there are substantial differences in the energy gain rates of the adsorbates that are related to the distinct electronic and phononic excitation times: The electrons drive heating at short times $(<1 \mathrm{ps})$, while the lattice promotes continued excitation at longer times. Finally, at $0.33 \mathrm{ML}$, we obtain the same desorption probability with the complete model and when only phonons are included, whereas no molecules are desorbed when only electronic excitations are allowed. Yet, our statistics is insufficient to conclusively disentangle the role of the different energy exchange channels in this particular case.

The simulations also allow us to determine what excitation mechanisms contribute to desorption from each specific adsorption site at $0.75 \mathrm{ML}$. We observe in Table I that when the excited phonons are neglected $\left(T_{e^{-}}\right.$ AIMDEF), desorption from the hollow sites is nearly eliminated, whereas desorption from the top site is only reduced around a factor 2 . In contrast, the percentage of molecules desorbing from the hollow sites when only phononic excitations are included ( $T_{l}$-AIMD) is approximately equal to that in the case in which both mechanisms are open $\left[\left(T_{e}, T_{l}\right)\right.$-AIMDEF $]$. These comparisons indicate that desorption from the hollow sites depends strongly on the excitation of the lattice nuclei. At the same time, we find that desorption from these sites driven by lattice excitation alone is 3 times less effective than when both excited electrons and phonons contribute. This shows that both electronic and phononic energy coupling cooperate to drive $\mathrm{CO}$ desorption in $\mathrm{Pd}(111)$ at $0.75 \mathrm{ML}$. Specifically, hot electrons efficiently couple energy into $\mathrm{CO}$ translational degrees of freedom while hot phonons reduce the CO-Pd bond strength by destabilizing the adsorbed $\mathrm{CO}$ from its equilibrium positions. The latter can be inferred by comparing the time evolution of the $\mathrm{CO}$ displacements obtained when $T_{l}$ is or not included in the simulations (top panels in Fig. 2). As a result, the hot electrons are more efficient in desorbing the phonon-induced unstable $\mathrm{CO}$ molecules. Figure 3 is a good example of how $T_{l}$ affects the $\mathrm{CO}$ trajectories at $0.75 \mathrm{ML}$. A similar CO destabilization is also observed at $0.33 \mathrm{ML}$ [26].

Finally, we analyze the importance of interadsorbate energy exchange at $0.75 \mathrm{ML}$ by performing simulations in which the Pd surface atoms and the $\mathrm{CO}$ adsorbed at the hollow sites are kept frozen $\left(T_{e}^{\mathrm{FHW}}\right.$-AIMDEF). These results are to be compared to those obtained within the

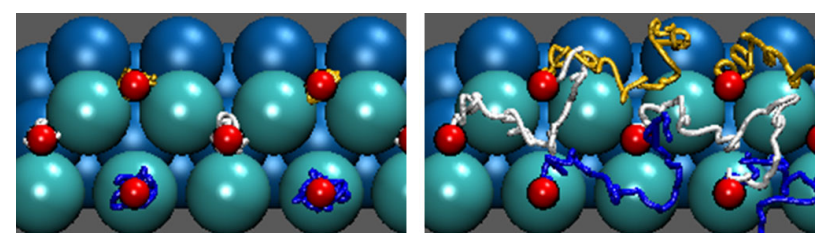

FIG. 3. Examples of typical CO trajectories in $T_{e}$-AIMDEF (left) and $\left(T_{e}, T_{l}\right)$-AIMDEF (right) simulations for $0.75 \mathrm{ML}$.

$T_{e}$-AIMDEF approach that allows excitation of the hollowsite molecules and the consequent interadsorbate energy exchange between top-site and hollow-site molecules. As shown in Table I, the top-CO desorption probability is reduced by a factor of 4 when the excitation of the hollow site molecules is neglected. This result demonstrates the importance of interadsorbate energy exchange at high coverage suggested previously on the basis that the nearest-neighbor separation at saturation is slightly less than the adsorbate van der Waals diameter [22]. The simulations enable us to extract information on the time evolution of this mechanism by comparing the mean kinetic energies of the nondesorbing top CO for the $T_{e}$-AIMDEF and $T_{e}^{\mathrm{FHW}}$-AIMDEF simulations (Fig. 2, left panel). At times below 1 ps both simulations give roughly the same energy gain, showing that at these short times the interadsorbate energy transfer is not active and, hence, the adsorbates only gain energy directly from the excited electronic system. However, the higher $\left\langle E_{\text {kin }}\right\rangle$ attained in the $T_{e}$-AIMDEF simulations at longer times highlights the importance of the interadsorbate energy exchange in promoting desorption. Thus our simulations support the desorption mechanism inferred from the experimental coverage dependence [22], namely, that fast electronic coupling to hollow site adsorbates enhances energy transfer to top-site adsorbates.

In summary, we have extended the AIMDEF method to include both time dependent electronic and phononic temperatures. This has allowed us to simulate femtosecond laser-induced reactions of adsorbates at surfaces by including both electronic and phononic excitations based on a two temperature model. Using these complete simulations that provide time and space resolution at the atomic scale, we are able to qualitatively reproduce and understand the strong coverage dependent desorption recently reported for $\mathrm{CO} / \mathrm{Pd}(111)$. The main reason for such dependence is the decrease of the $\mathrm{CO}$ desorption barriers with coverage. Additionally, for large coverages we have demonstrated the importance of interadsorbate energy exchange that enhances desorption, and, surprisingly, the complementary contribution of both the excited electrons and phonons that has largely been neglected up to now. Detailed simulations show that hot phonons reduce the adsorbate-surface binding by bringing $\mathrm{CO}$ out of the adsorption well, considerably increasing the efficiency with which hot electrons drive desorption at $0.75 \mathrm{ML}$. This cooperative effect seems rather 
general and, hence, we expect it to be present in other systems, particularly at high surface temperatures. In this respect, it is worth mentioning that recent calculations on the vibrational lifetimes of adsorbates on metals also indicate the importance of the electron-excited phonons in this related process $[71,72]$.

M. A. and J. I. J. acknowledge financial support by the Gobierno Vasco-UPV/EHU project IT1246-19, and the Spanish Ministerio de Economía y Competitividad [Grant No. FIS2016-76471-P (AEI/FEDER, UE)]. The contributions of N.C. and S. Y.H. were supported by the U.S. Department of Energy, Office of Science, Office of Basic Energy Sciences, Chemical Sciences, Geosciences, \& Biosciences Division, Condensed Phase and Interfacial Molecular Science Program, under Contract No. DESC0012704. Computational resources were provided by the DIPC computing center.

*maite.alducin@ehu.eus

†nicholas@bnl.gov

*perpc80@gmail.com

josebainaki.juaristi@ehu.eus

[1] C. Frischkorn and M. Wolf, Chem. Rev. 106, 4207 (2006).

[2] H. Guo, P. Saalfrank, and T. Seideman, Prog. Surf. Sci. 62, 239 (1999).

[3] P. Saalfrank, Chem. Rev. 106, 4116 (2006).

[4] R. R. Cavanagh, D. S. King, J. C. Stephenson, and T. F. Heinz, J. Phys. Chem. 97, 786 (1993).

[5] F. Budde, T. F. Heinz, M. M. T. Loy, J. A. Misewich, F. de Rougemont, and H. Zacharias, Phys. Rev. Lett. 66, 3024 (1991).

[6] J. A. Misewich, T. F. Heinz, and D. M. Newns, Phys. Rev. Lett. 68, 3737 (1992).

[7] D. G. Busch, S. Gao, R. A. Pelak, M. F. Booth, and W. Ho, Phys. Rev. Lett. 75, 673 (1995).

[8] D. N. Denzler, C. Frischkorn, C. Hess, M. Wolf, and G. Ertl, Phys. Rev. Lett. 91, 226102 (2003).

[9] F. Fournier, W. Zheng, S. Carrez, H. Dubost, and B. Bourguignon, J. Chem. Phys. 121, 4839 (2004).

[10] M. Bonn, S. Funk, C. Hess, D. N. Denzler, C. Stampfl, M. Scheffler, M. Wolf, and G. Ertl, Science 285, 1042 (1999).

[11] S. Funk, M. Bonn, D. N. Denzler, C. Hess, M. Wolf, and G. Ertl, J. Chem. Phys. 112, 9888 (2000).

[12] R. Scholz, G. Floß, P. Saalfrank, G. Füchsel, I. Lončarić, and J. I. Juaristi, Phys. Rev. B 94, 165447 (2016).

[13] I. Lončarić, M. Alducin, P. Saalfrank, and J. I. Juaristi, Phys. Rev. B 93, 014301 (2016).

[14] M. Blanco-Rey, J. I. Juaristi, R. Díez Muiño, H. F. Busnengo, G. J. Kroes, and M. Alducin, Phys. Rev. Lett. 112, 103203 (2014).

[15] P. Saalfrank, J. I. Juaristi, M. Alducin, M. Blanco-Rey, and R. Díez Muiño, J. Chem. Phys. 141, 234702 (2014).

[16] D. Novko, M. Blanco-Rey, J. I. Juaristi, and M. Alducin, Phys. Rev. B 92, 201411(R) (2015).

[17] D. Novko, M. Blanco-Rey, M. Alducin, and J. I. Juaristi, Phys. Rev. B 93, 245435 (2016).
[18] D. Novko, M. Blanco-Rey, J. I. Juaristi, and M. Alducin, Nucl. Instrum. Methods Phys. Res., Sect. B 382, 26 (2016).

[19] D. Novko, I. Lončarić, M. Blanco-Rey, J. I. Juaristi, and M. Alducin, Phys. Rev. B 96, 085437 (2017).

[20] J. I. Juaristi, M. Alducin, and P. Saalfrank, Phys. Rev. B 95, 125439 (2017).

[21] H. Xin et al., Phys. Rev. Lett. 114, 156101 (2015).

[22] S.-Y. Hong, P. Xu, N. R. Camillone, M. G. White, and N. Camillone III, J. Chem. Phys. 145, 014704 (2016).

[23] P. Szymanski, A. L. Harris, and N. Camillone, J. Chem. Phys. 126, 214709 (2007).

[24] W. G. Roeterdink, E. H. G. Backus, A. W. Kleyn, and M. Bonn, Phys. Rev. Lett. 93, 249601 (2004).

[25] L. Cai, X. Xiao, and M. M. T. Loy, J. Chem. Phys. 115, 9490 (2001).

[26] See Supplemental Material at http://link.aps.org/ supplemental/10.1103/PhysRevLett.123.246802, which additionally includes Refs. [27-43], for more details on the theoretical framework, computational methods, and dynamics.

[27] M. Lisowski, P. Loukakos, U. Bovensiepen, J. Stähler, C. Gahl, and M. Wolf, Appl. Phys. A 78, 165 (2004).

[28] T. Avanesian and P. Christopher, J. Phys. Chem. C 118, 28017 (2014).

[29] W. S. Fann, R. Storz, H. W. K. Tom, and J. Bokor, Phys. Rev. B 46, 13592 (1992).

[30] A. P. Miiller and B. N. Brockhouse, Phys. Rev. Lett. 20, 798 (1968).

[31] I. Lončarić, M. Alducin, P. Saalfrank, and J. I. Juaristi, Nucl. Instrum. Methods Phys. Res., Sect. B 382, 114 (2016).

[32] F. L. Hirshfeld, Theor. Chim. Acta 44, 129 (1977).

[33] S. P. Rittmeyer, J. Meyer, J. I. Juaristi, and K. Reuter, Phys. Rev. Lett. 115, 046102 (2015).

[34] E. Zaremba, J. H. Rose, L. M. Sander, and H. B. Shore, J. Phys. F 7, 1763 (1977).

[35] P. M. Echenique, R. M. Nieminen, and R. H. Ritchie, Solid State Commun. 37, 779 (1981).

[36] X. Luo, B. Jiang, J. I. Juaristi, M. Alducin, and H. Guo, J. Chem. Phys. 145, 044704 (2016).

[37] J. P. Perdew, K. Burke, and M. Ernzerhof, Phys. Rev. Lett. 77, 3865 (1996).

[38] I. Lončarić, M. Alducin, J. I. Juaristi, and D. Novko, J. Phys. Chem. Lett. 10, 1043 (2019).

[39] P. E. Blöchl, Phys. Rev. B 50, 17953 (1994).

[40] G. Kresse and D. Joubert, Phys. Rev. B 59, 1758 (1999).

[41] H. J. Monkhorst and J. D. Pack, Phys. Rev. B 13, 5188 (1976).

[42] M. Methfessel and A. T. Paxton, Phys. Rev. B 40, 3616 (1989).

[43] G. Mills, H. Jónsson, and G. K. Schenter, Surf. Sci. 324, 305 (1995).

[44] C. Springer, M. Head-Gordon, and J. C. Tully, Surf. Sci. 320, L57 (1994).

[45] C. Springer and M. Head-Gordon, Chem. Phys. 205, 73 (1996).

[46] S. I. Anismov, B. L. Kapeliovich, and T. L. Perel'man, Zh. Eksp. Teor. Fiz. 66, 776 (1974) [Sov. Phys. JETP 39, 375 (1974)].

[47] T. Vazhappilly, T. Klamroth, P. Saalfrank, and R. Hernandez, J. Phys. Chem. C 113, 7790 (2009). 
[48] G. Füchsel, T. Klamroth, J. C. Tremblay, and P. Saalfrank, Phys. Chem. Chem. Phys. 12, 14082 (2010).

[49] G. Füchsel, T. Klamroth, S. Monturet, and P. Saalfrank, Phys. Chem. Chem. Phys. 13, 8659 (2011).

[50] P. D. Johnson, D. A. Wesner, J. W. Davenport, and N. V. Smith, Phys. Rev. B 30, 4860 (1984).

[51] J. Rogozik and V. Dose, Surf. Sci. 176, L847 (1986).

[52] P. D. Johnson and S. L. Hulbert, Phys. Rev. B 35, 9427 (1987).

[53] E. T. Jensen, R. E. Palmer, R. F. Willis, I. R. Collins, and P. T. Andrews, Vacuum 38, 353 (1988).

[54] J. I. Juaristi, M. Alducin, R. Díez Muiño, H. F. Busnengo, and A. Salin, Phys. Rev. Lett. 100, 116102 (2008).

[55] M. Alducin, R. Díez Muiño, and J. I. Juaristi, Prog. Surf. Sci. 92, 317 (2017).

[56] S. Nosé, J. Chem. Phys. 81, 511 (1984).

[57] W. G. Hoover, Phys. Rev. A 31, 1695 (1985).

[58] H. Ohtani, M. V. Hove, and G. Somorjai, Surf. Sci. 187, 372 (1987).

[59] W. K. Kuhn, J. Szanyi, and D. Goodman, Surf. Sci. 274, L611 (1992).

[60] T. Gießel, O. Schaff, C. Hirschmugl, V. Fernandez, K.-M. Schindler, A. Theobald, S. Bao, R. Lindsay, W. Berndt, A. Bradshaw, C. Baddeley, A. Lee, R. Lambert, and D. Woodruff, Surf. Sci. 406, 90 (1998).
[61] S. Surnev, M. Sock, M. Ramsey, F. Netzer, M. Wiklund, M. Borg, and J. Andersen, Surf. Sci. 470, 171 (2000).

[62] M. Rose, T. Mitsui, J. Dunphy, A. Borg, D. Ogletree, M. Salmeron, and P. Sautet, Surf. Sci. 512, 48 (2002).

[63] D. Loffreda, D. Simon, and P. Sautet, Surf. Sci. 425, 68 (1999).

[64] C. Fan and W.-D. Xiao, Comput. Theor. Chem. 1004, 22 (2013).

[65] G. Kresse and J. Furthmüller, Comput. Mater. Sci. 6, 15 (1996).

[66] G. Kresse and J. Furthmüller, Phys. Rev. B 54, 11169 (1996).

[67] M. Dion, H. Rydberg, E. Schröder, D. C. Langreth, and B. I. Lundqvist, Phys. Rev. Lett. 92, 246401 (2004).

[68] P. Lazić, M. Alaei, N. Atodiresei, V. Caciuc, R. Brako, and S. Blügel, Phys. Rev. B 81, 045401 (2010).

[69] P. Szymanski, A. Harris, and N. Camillone III, Surf. Sci. 601, 3335 (2007).

[70] K.-I. Inoue, K. Watanabe, T. Sugimoto, Y. Matsumoto, and T. Yasuike, Phys. Rev. Lett. 117, 186101 (2016).

[71] D. Novko, M. Alducin, and J. I. Juaristi, Phys. Rev. Lett. 120, 156804 (2018).

[72] D. Novko, J. C. Tremblay, M. Alducin, and J. I. Juaristi, Phys. Rev. Lett. 122, 016806 (2019). 